\title{
An ioMRI-assisted case of cervical intramedullary diffuse glioma resection
}

Zafer Orkun Toktas, ${ }^{1,2}$

Baran Yilmaz,' Murat Șakir Ekși, ${ }^{1,3}$ Lei Wang, ${ }^{2,4}$ Akin Akakin, ${ }^{1}$ Yasin Yener, ${ }^{5}$ Murat Konakcı, ${ }^{6}$ Emre Ayan, ${ }^{7}$ Turker Kılıc,' Deniz Konya, ${ }^{1,2}$ Yang D Teng ${ }^{2,4}$

'Department of Neurosurgery, Bahçeșehir University Medical Faculty, Istanbul, Turkey; ${ }^{2}$ Departments of Physical Medicine and Rehabilitation and Neurosurgery, Harvard Medical School, Boston, MA, USA; ${ }^{3}$ Department of Orthopedic Surgery, University of California at San Francisco, San Francisco, CA, USA; ${ }^{4}$ Division of Spinal Cord Injury Research, Veterans Affairs Healthcare System, Boston, MA, USA; ${ }^{5}$ Department of Anesthesiology, Göztepe Medikal Park Hospital, Istanbul, Turkey; ${ }^{6}$ Department of Anesthesiology, Bahçeșehir University Medical Faculty, Istanbul, Turkey; ${ }^{7}$ Department of Radiology, Göztepe Medical Park, Istanbul, Turkey

Correspondence: Yang D Teng Departments of Physical Medicine and Rehabilitation and Neurosurgery, Harvard Medical School, 300 Ist Avenue, BLD-79/96, Charlestown, MA 02129, USA

Tel + I 6179526172

$\mathrm{Fax}+\mathrm{I} 6179526060$

Email yang_teng@hms.harvard.edu

Zafer Orkun Toktas

Department of Neurosurgery, Bahçeșehir University Medical Faculty, Istanbul,

Turkey

Tel +905323078489

Email drzafertoktas@gmail.com
This article was published in the following Dove Press journal: Cancer Management and Research

Purpose: To date, application of intraoperative magnetic resonance imaging (ioMRI) to enhance surgical quality for spinal intramedullary neoplastic lesions has been rarely reported. Moreover, in developing countries or regions, ioMRI accessibility remains very limited. This report describes a technology design of high-field ioMRI accessible for multioperation rooms via a case presentation of an imaging-assisted surgical excision of human cervical spinal cord diffuse glioma.

Patient and methods: The patient was a 44-year-old woman with symptomatic and progressive C2-5 intramedullary diffuse glioma (IDG). Our ioMRI system was designed and arranged with accessibility to multiple operation rooms, which was used to assure more complete spinal cord or brain tumor removal. The intraoperational diagnostic aspects and the system setup technical details are presented for future applications of the system in hospitals where a designated ioMRI suite is not available.

Results: After a conventionally defined complete removal of C2-C5 IDG using a well-established surgical approach, ioMRI examination was able to detect residual tumor tissues that were indistinguishable under the surgical microscope. The IDG clusters were subsequently excised. The operation regimen resulted in a gross total elimination of the tumor, which enabled the patient to show very satisfactory postsurgery recovery and prognosis.

Conclusion: ioMRI-assisted surgical removal of cervical spinal cord diffuse glioma should be systematically developed and applied to enhance therapeutic efficacy. The reported logistic flow of operating room tasks and imaging technical management are innovative for performing the tumor removal procedures in hospitals where designated ioMRI surgical suites do not exist. Critically, we emphasize implementation of stringent quality control measures for patient transportation safety and contamination prevention in establishing and maintaining such a system.

Keywords: intraoperative imaging, spine, spinal cord, glioma, residual tumor, decompression

\section{Introduction}

Intraoperative magnetic resonance imaging (ioMRI) has been increasingly used for improving efficacy of surgical treatment, particularly for intracranial neurosurgical procedures. ${ }^{1}$ Tangible clinical reports have demonstrated that effective use of ioMRI systems helps to augment the efficiency of pathological locus targeting and tumoral removal..$^{2-4}$ However, establishing standard ioMRI setting in a totally designated operation room (OR) equipped with titanium MRI-safe instruments/tools is an exceedingly expensive endeavor, which has not been readily available for hospitals in developing countries or regions. Therefore, despite highly positive outcomes and recommendations from the initial exploration of ioMRI use in spine surgeries, ${ }^{5}$ judicious application of this technology to enhance surgical outcome quality for spinal intramedullary neoplastic lesions has been very rarely reported to date (Table S1) ${ }^{6}$ 
Intramedullary spinal cord tumors (IMSCT) comprise about $2 \%-4 \%$ of all central nervous system tumors, representing approximately $20 \%$ of spine tumors in adults and up to $35 \%$ of spine tumors in children. ${ }^{7}$ Surgery of the cervical intramedullary glia-origin tumors presents an extraordinary challenge due to concentrated neurobiological centers governing vital functions in the spinal cord segments. The completeness of surgical resection has been determined as a key factor for determining survival prognosis and as a main predictor of long-term outcomes. ${ }^{89}$ Here, we report an ioMRI-assisted surgical protocol designed for a cervical intramedullary diffuse glioma case. The paper is focused on assessing the creative use of the ioMRI equipment for resecting IMSCT, as well as its advantages, disadvantages, and safety issues.

\section{Clinical case and methods Patient}

A previously healthy 44-year-old woman presented with intermittent episodes of bilateral upper limb numbness over 8 months with worsening symptoms associated with bilateral hand clumsiness and unsteady gait in the past month. She denied having any sphincter dysfunction. Her neurological function status was Nurick grade III. Cervical MRI scan was prescribed for detecting possible pathological conditions.

\section{Diagnosis, assessment, and treatment plan}

The patient's general physical examination was unremarkable. The pathologic findings on neurological examination were as follows: spastic quadriparesis that was most prominent on the left side, hypoesthesia mainly between C5 and T1, exaggerated deep tendon reflexes on the left side limbs, and a positive Babinski's sign in the left foot. There was reduced vibration and position sense in the left toe and ankle. Rectal tone and sensation were both normal. The patient also exhibited Romberg's sign and spastic-ataxic type gait disturbances. T2-weighted MRI examination of her cervical spine revealed a hyperintense C2-C5 intramedullary lesion with spinal cord expansion and edema extending rostrally into the medulla and caudally to the thoracic spinal cord (Figure 1). There was no contrast enhancement. We therefore decided to perform microsurgical tumor resection with the aid of a unique imaging protocol that allowed application of high-field ioMRI in combination with neurophysiological monitoring of somatosensory and motor-evoked potentials (MEPs). For the ioMRI T2WI-detected hyperintensity zone, the area was first explored under microscopic guidance. Following tumor confirmation, the resection was performed under stringent control of MEP neuromonitoring. The design prevented us from mistaking operation-induced hyperintensities as tumor masses. Our approach, with enhanced safety measures for patient protection, augmented surgical completeness of IMSCT resection in a multiple operating room (OR) setting that did not have a designated ioMRI operation suite.

\section{Multi-OR ioMRI concept}

At Bahcesehir University, Goztepe Medical Park Hospital, any of the six operating rooms in our surgical theater is designed to have access to the ioMRI (MRW450) system between surgical procedures or stages (Figure 2). Implementation of this novel concept permits that each OR can be assigned for its routine operations with prephased access to ioMRI assistance. Furthermore, the use of MR compatible headrest-head coil unit (General Electric Healthcare, Chicago, IL, USA) allows the patient to be imaged directly at planned procedure time point. The surgical tables (Maquet GmbH, Rastatt, Germany) used in our OR are adapted to be interchangeable with an MRI compatible transfer table that enables transient transportation of patient to MRI gantry under general anesthesia. In the present case, the patient was positioned and fixed in prone position for posterior midline approach to the cervical intramedullary region. The thorax and pelvis of the patient were supported by silicon pads. At time of transfer, the operative field was covered with a new drape, coverings beyond the operative field were removed, and the patient was transferred through the connection door to the ioMRI system. Then, the MRI head coil was mounted on the headrest. After ioMRI scanning, the patient was brought back to the operating table, with a new drape being placed on the operative site followed by a general redraping. Images were processed and displayed immediately after acquisition in the monitoring room (Figure 3). Each step was controlled manually, and the members of the surgical team (ie, the primary surgeon and assistant surgeon, anesthesiologist, and radiologist) supervised the transfer to ensure patient safety. The primary neurosurgeon and radiologist interpreted the ioMRI results before refinement microsurgery was performed to ensure a complete removal of the neoplastic mass (Figure 3; Supplementary video).

\section{Tissue bank}

The surgically removed tumoral tissue was preserved in The Bahcesehir University Tissue Bank (BUTB). BUTB maintains a tissue database with links to our general clinicopathological data storage. The tissue sample was snap-frozen 


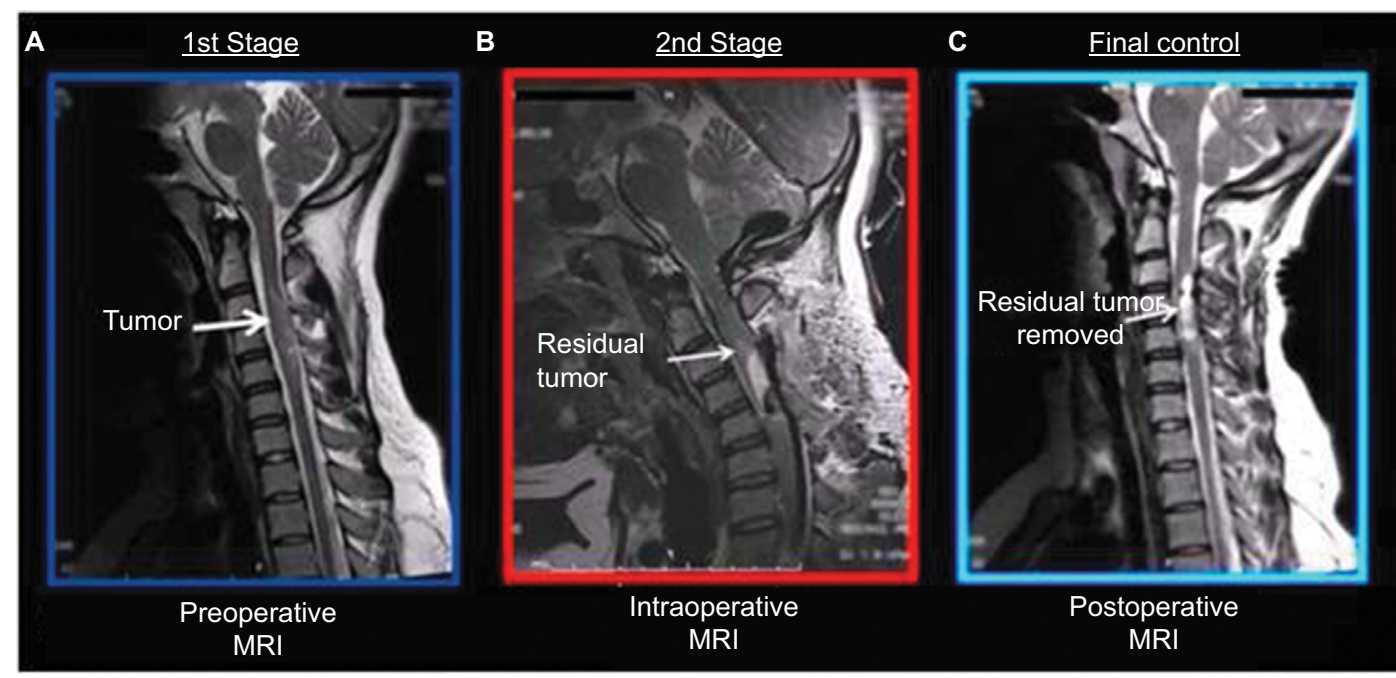

Figure I (A) T2-weighted MRI examination of the cervical spine revealed a hyperintense C2-C5 intramedullary lesion with surrounding spinal cord tissue expansion and edema. The overall pathological volume extended rostrally into the medulla and caudally into the thoracic segments of the spinal cord. (B) Based on the evaluation of the intraoperationally obtained images, a targeted surgical plan was formed. (C) The last image showed that the following up microsurgical procedures ensured an essentially complete removal of the residual tumor tissues.

Abbreviation: MRI, magnetic resonance imaging.

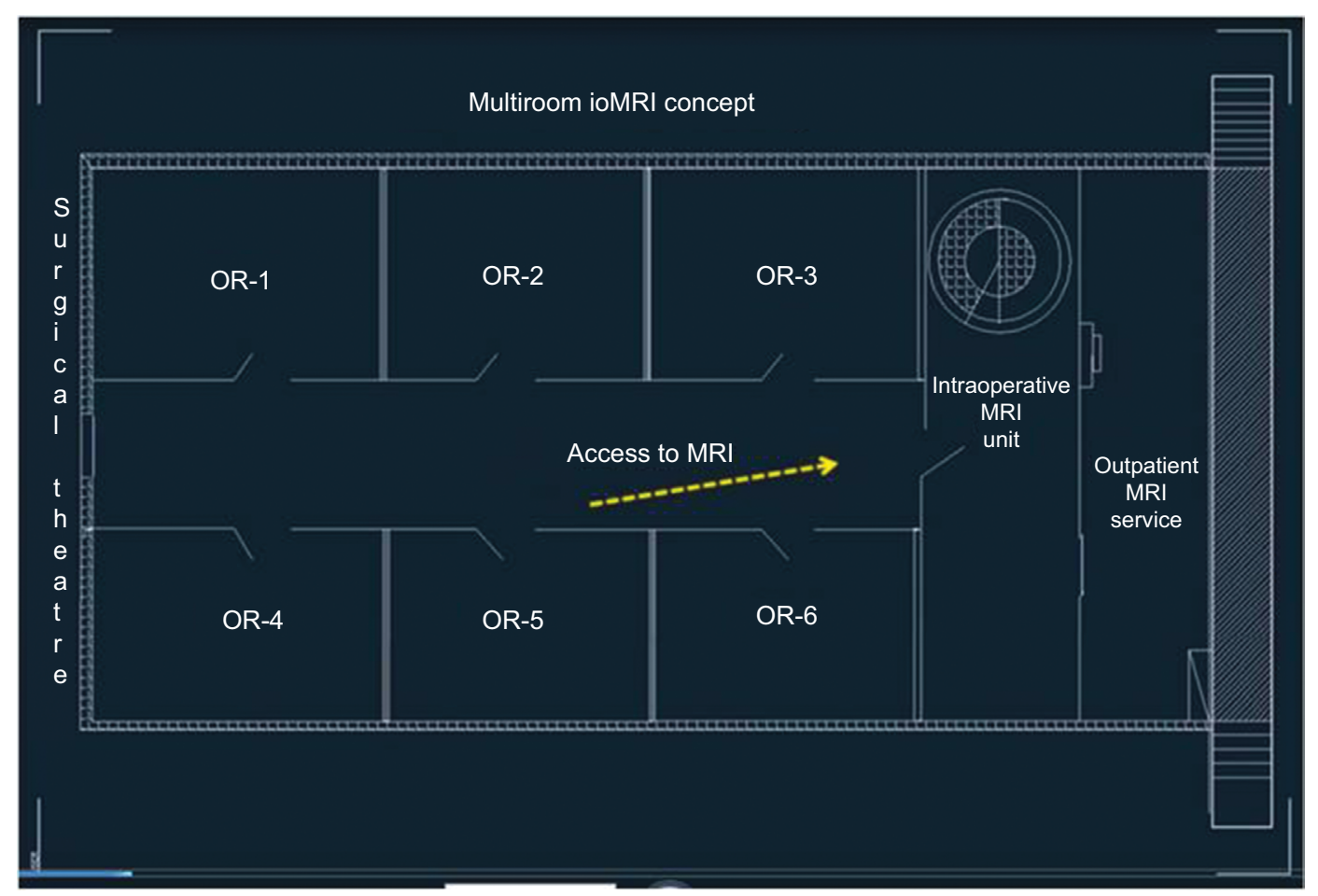

Figure 2 In our surgical theater, each OR is assigned with capacities for both its routine surgical occupation and prephased or interphased accesses to the ioMRI imaging assistances.

Abbreviations: OR, operating room; ioMRI, intraoperative magnetic resonance imaging.

in liquid nitrogen immediately after surgical removal and subsequently transferred from the OR to the tissue bank's liquid nitrogen bank for long-term storage and future analytical studies.

\section{Results}

Surgery was started after positioning of the needles for neurophysiological monitoring of somatosensory and MEPs. The neoplastic lesion was reached via posterior median approach 


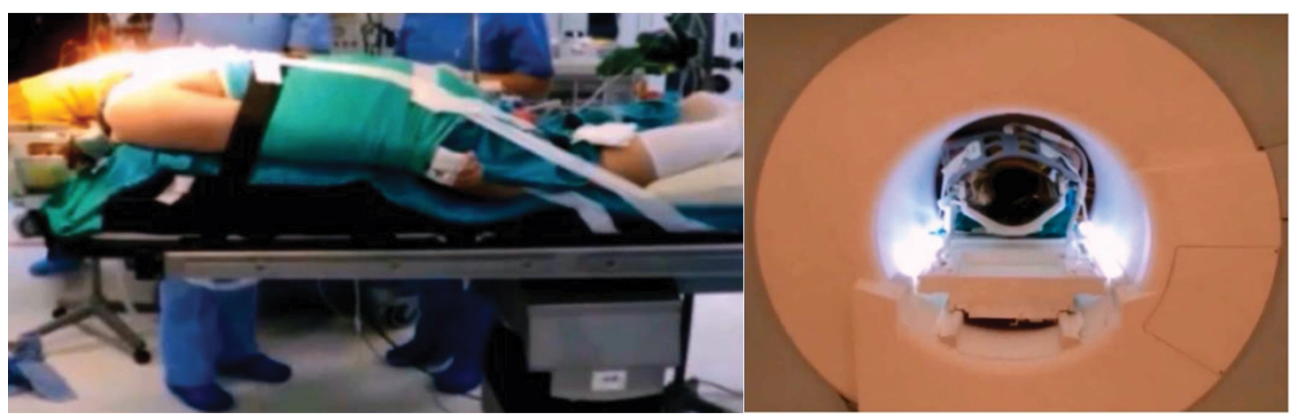

Figure 3 ioMRI system enhances neurosurgical procedures by displaying highly accurate, real-time digital images of the cervical spine during surgery. Abbreviations: ioMRI, intraoperative magnetic resonance imaging.

to the cervical spine and spinal cord. After the primary neurosurgeon microscopically achieved a satisfactory resection by the best conventional neurosurgical judgment, the patient was transiently transported for receiving an ioMRI evaluation per procedures detailed in the "Methods". MRI outcome was jointly assessed with the onsite radiologist. They identified a small volume of residual tumor located toward the cranial end of the surgical field that was subsequently and accordingly removed as confirmed by MRI diagnosis afterwards. Postsurgery pathological assessment defined the tumor as low-grade astrocytoma based on World Health Organization classifications. With the patient's written consent, the removed tissue was kept at BUTB. The patient recovered without any complication and remained well in our last following up 6 months later.

During the whole operation, somatosensory and MEPs remained stable. No anesthesia-related problems or perioperative complications were encountered. The interprocedural ioMRI evaluation added about $40 \%$ more anesthesia time compared with a conventional approach without neural imaging assistance. Based on published data, we deemed the benefits of the enhanced completeness of tumor resection overweighed potential drawbacks of the limited increase of anesthesia period.

\section{Discussion}

In our institution, the multi-OR ioMRI concept and design, an upgrade from the so-called "shared-resource" system enables interprocedural imaging assistance for microsurgical resection of intramedullary diffuse neoplasm in high risk regions such as cervical spinal cord. It permits allocating the highfield MRI unit for outpatient diagnostic use when imaging is not needed intraoperatively., ${ }^{3,10}$ In this setting, the interroom gating is provided by a magnetically shielded whole piece door and patient transportation via an MRI-safe transfer table. Under this system, the OR and MRI room can be used either combinatorially or independently. Therefore, the system is cost-efficient for regions and countries where establishing commercially engineered OR equipped with a designated ioMRI unit (eg, Brain Suite) is not financially feasible.

Although there are a number of reports on ioMRI-assisted brain operations, ${ }^{1-4,11,12}$ only very limited cases have been published regarding the use of ioMRI systems in spinal surgery, ${ }^{1,6,13}$ in spite of the initial favorable evaluation and strong recommendation. ${ }^{5}$ For example, Giordano et $\mathrm{al}^{6}$ described two patients for whom operations were performed in a Brain Suite, a dedicated radiofrequency-shielded operating room equipped with a high-field 1.5 T MR imager., ${ }^{6,11}$ Another group performed a posterior approach for intramedullary tumor biopsy with the coil and setting standardly deployed for intracranial surgery. ${ }^{1}$ Additional reports on the use of various ioMRI systems in spinal surgery concerned treatments of nontumoral pathologies, including discectomy, posterior decompression for degenerative diseases, and anterior decompression and fusion for cervical spondylotic myelopathy. ${ }^{5,13,14}$ Thus, to our best knowledge, we performed one of the first cases in the literature of an intramedullary tumor surgery with ioMRI; through this report, we introduced a novel multi-OR ioMRI concept and an effective ioMRI system that incorporated both high-field MR imaging and a shared-resource design for spinal neurosurgery.

For this case, we did not have any software of diffusion tensor imaging to assess possible tumor encasing of white matter axons. ${ }^{15}$ Thus, maximum feasible tumor resection was performed, based on intraoperative MEP neuromonitoringdefined safe surgery zones. Our MRI-safe transfer table, exchangeable operating table, and headrest coil had certain limitations that, depending on specific needs of some patients, might affect practicality of performing certain spinal procedures. For instance, patients with broad shoulders and short necks could not be readily positioned in the aforementioned system. We are designing additional equipment for increased physical adaptation capacity. We emphasize that in order to 
maximize quality control under a shared-resource design, the members of the surgical team (i.e., primary, assistant surgeons and nurses plus onsite anesthesiologist and radiologist) should have a predelegated responsibility sharing plan. All should participate and/or supervise task execution to secure achieving stage-specific goals. Lastly, OR-related infection has been a known risk factor, ${ }^{16,17}$ but our experience showed that carefully applied sterile draping before and after each procedure transition and thorough MRI room sterilization before and after each intraoperational visit could help to avoid wound contamination. To establish the costeffective approach for the ioMRI access we reported, there are serious training and organization endpoints that the team members must accomplish. These include safety insurance for the patients during transportation, establishing a reliable collaborative OR workflow, and sterile maintenance for the patient's stabilization instruments, OR, transportation corridor, and ioMRI room. All, in fact, can be achieved through a systematically designed quality control system with periodical performance validations.

The ioMRI technique shows special value in delineating the tumor-spinal cord interface where normal anatomy has been deformed and surgical microscopic discrimination between tumor and cord tissue is mostly infeasible, especially in larger tumor cases. Encouragingly, less expensive intraoperative imaging navigation systems recently became available, which has shown benefits for doing more precise resections of intracranial gliomas. ${ }^{17-19}$ Theoretically, the use of such a navigation system to guide a surgical resection plan that is based on an MRI-defined tumor topology profile could also be an improved approach for hospitals where a designated ioMRI suite is not available. Systematical studies to test the efficacy of such combinatorial applications should be carried out in the future to maximize surgical efficacy and help with development of targeted treatments for intramedullary diffuse glioma. ${ }^{20}$

\section{Conclusion}

The present case demonstrates that a unique ioMRI system, which has a distinct capability to sequentially serve multiple patients during their surgeries and carries excellent value to eliminate residual volume of cervical spinal cord diffuse glioma. The operation was simultaneously supported by neurophysiological monitoring, an important technical enhancement that is not practicable in current Brain Suite or Brain Suite-like settings. We highlight the importance of sterile handling and safety standards in patient transportation, and acknowledge that well-designed longitudinal prospec- tive studies are needed in the future to further validate the beneficial impact of similar systems.

\section{Ethics approval}

Written informed consent has been provided by the patient to have the case details and any accompanying images published.

\section{Acknowledgment}

Work at Dr Teng's Lab was additionally supported by Department of Defense, Veterans Affairs Research \& Development, The Center for the Advancement of Science in Space and The National Aeronautics and Space Administration, and Gordon Project to Treat Clinical Paralysis at Harvard Medical School and Spaulding Rehabilitation Hospital.

\section{Author contributions}

The patient was operated by DK. ZOT, BY, DK, TK, and YDT designed the overall study. ZOT, BY, DK, and YDT organized the data and drafted the manuscript. YDT finalized the paper with all authors. All authors contributed toward data analysis, drafting and critically revising the paper and agree to be accountable for all aspects of the work.

\section{Disclosure}

The authors report no conflicts of interest in this work.

\section{References}

1. Hall WA, Truwit CL. Intraoperative magnetic resonance imaging. Acta Neurochir Suppl. 2011;109:119-129.

2. Nimsky C, Fujita A, Ganslandt O, von Keller B, Fahlbusch R. Volumetric assessment of glioma removal by intraoperative high-field magnetic resonance imaging. Neurosurgery. 2004;55(2):358-371.

3. Pamir MN, Ozduman K, Dinçer A, Yildiz E, Peker S, Ozek MM. First intraoperative, shared-resource, ultrahigh-field 3-Tesla magnetic resonance imaging system and its application in low-grade glioma resection. J Neurosurg. 2010;112(1):57-69.

4. Fahlbusch R, Keller B, Ganslandt O, Kreutzer J, Nimsky C. Transsphenoidal surgery in acromegaly investigated by intraoperative highfield magnetic resonance imaging. Eur J Endocrinol. 2005;153(2): 239-248.

5. Woodard EJ, Leon SP, Moriarty TM, Quinones A, Zamani AA, Jolesz FA. Initial experience with intraoperative magnetic resonance imaging in spine surgery. Spine. 2001;26(4):410-417.

6. Giordano M, Gerganov VM, Metwali H, et al. Feasibility of cervical intramedullary diffuse glioma resection using intraoperative magnetic resonance imaging. Neurosurg Rev. 2013;37:139-146.

7. Raco A, Esposito V, Lenzi J, Piccirilli M, Delfini R, Cantore G. Longterm follow-up of intramedullary spinal cord tumors: a series of 202 cases. Neurosurgery. 2005;56(5):972-981.

8. Klekamp J. Treatment of intramedullary tumors: analysis of surgical morbidity and long-term results. J Neurosurg Spine. 2013;19(1) $12-26$.

9. Kothbauer KF. Neurosurgical management of intramedullary spinal cord tumors in children. Pediatr Neurosurg. 2007;43(3):222-235. 
10. Steinmeier R, Fahlbusch R, Ganslandt O, et al. Intraoperative magnetic resonance imaging with the magnetom open scanner: concepts, neurosurgical indications, and procedures: a preliminary report. Neurosurgery. 1998;43(4):739-747; discussion 747-748.

11. Nimsky C, Ganslandt O, Fahlbusch R. Comparing 0.2 tesla with 1.5 tesla intraoperative magnetic resonance imaging analysis of setup, workflow, and efficiency. Acad Radiol. 2005;12:1065-1079.

12. Hatipoğlu MA, Prabhu S, Weinberg J, Shah K, Sawaya R. Surgical treatment of supplementary motor area lesions. Turk Neurosurg. 2009;19(3): 306-307.

13. Mastronardi L, Elsawaf A, Roperto R, et al. Prognostic relevance of the postoperative evolution of intramedullary spinal cord changes in signal intensity on magnetic resonance imaging after anterior decompression for cervical spondylotic myelopathy. J Neurosurg Spine. 2007;7(6):615-622.

14. Verheyden P, Katscher S, Schulz T, Schmidt F, Josten C. Open MR imaging in spine surgery: experimental investigations and first clinical experiences. Eur Spine J. 1999;8(5):346-353.
15. Liu X, Tian W, Chen H, et al. Advanced neuroimaging in the evaluation of spinal cord tumors and tumor mimics: diffusion tensor and perfusionweighted imaging. Semin Ultrasound CT MR. 2017;38(2):163-175.

16. Bohinski RJ, Kokkino AK, Warnick RE, et al. Glioma resection in a sharedresource magnetic resonance operating room after optimal image-guided frameless stereotactic resection. Neurosurgery. 2001;48(4):731-744.

17. Mert A, Kiesel B, Wöhrer A, et al. Introduction of a standardized multimodality image protocol for navigation-guided surgery of suspected low-grade gliomas. Neurosurg Focus. 2015;38(1):E4.

18. Mert A, Buehler K, Sutherland GR, et al. Brain tumor surgery with 3-dimensional surface navigation. Neurosurgery. 2012;71(2 Suppl Operative):ons286-ons295.

19. Tanaka Y, Nariai T, Momose T, et al. Glioma surgery using a multimodal navigation system with integrated metabolic images. J Neurosurg. 2009;110(1):163-172.

20. Ropper AE, Zeng X, Haragopal H, et al. Targeted treatment of experimental spinal cord glioma with dual gene-engineered human neural stem cells. Neurosurgery. 2016;79(3):481-491.
Cancer Management and Research

\section{Publish your work in this journal}

Cancer Management and Research is an international, peer-reviewed open access journal focusing on cancer research and the optimal use of preventative and integrated treatment interventions to achieve improved outcomes, enhanced survival and quality of life for the cancer patient. The manuscript management system is completely online and includes
Dovepress

a very quick and fair peer-review system, which is all easy to use. Visit $\mathrm{http}: / / \mathrm{www}$.dovepress.com/testimonials.php to read real quotes from published authors. 